\title{
Atypical categorization in children with high-functioning autism spectrum disorder
}

\author{
Barbara A. Church and Maria S. Krauss \\ University at Buffalo, State University of New York, Buffalo, New York \\ Christopher Lopata \\ Institute for Autism Research, Canisius College, Buffalo, New York \\ JENNIFER A. TOOMEY \\ Summit Educational Resources, Getzville, New York \\ Marcus L. Thomeer \\ Institute for Autism Research, Canisius College, Buffalo, New York \\ AND
}

\author{
Mariana V. Coutinho, Martin A. Volker, and Eduardo Mercado III \\ University at Buffalo, State University of New York, Buffalo, New York
}

\begin{abstract}
Children with autism spectrum disorder process many perceptual and social events differently from typically developing children, suggesting that they may also form and recognize categories differently. We used a dot pattern categorization task and prototype comparison modeling to compare categorical processing in children with high-functioning autism spectrum disorder and matched typical controls. We were interested in whether there were differences in how children with autism use average similarity information about a category to make decisions. During testing, the group with autism spectrum disorder endorsed prototypes less and was seemingly less sensitive to differences between to-be-categorized items and the prototype. The findings suggest that individuals with high-functioning autism spectrum disorder are less likely to use overall average similarity when forming categories or making categorical decisions. Such differences in category formation and use may negatively impact processing of socially relevant information, such as facial expressions. A supplemental appendix for this article may be downloaded from http://pbr.psychonomic-journals.org/content/supplemental.
\end{abstract}

Individuals with autism spectrum disorder (ASD) have enhanced perceptual discrimination but deficits in processing configural information. They show accelerated learning on some tasks (e.g., O'Riordan \& Plaisted, 2001) but distinct deficits in perceptual learning and generalizing to novel situations (Klinger \& Dawson, 2001; Plaisted, O'Riordan, \& Baron-Cohen, 1998). Many competing theories have been developed to explain these findings (e.g., Iarocci \& McDonald, 2006; Just, Cherkassky, Keller, \& Minshew, 2004; McClelland, 2000). Three of the most influential - weak central coherence (Happe \& Frith, 2006), enhanced perceptual functioning (Mottron, Dawson, Soulieres, Hubert, \& Burack, 2006), and reduced perceptual similarity (Plaisted, 2001) - converge on the idea that individuals with ASD form hyperspecific representations that affect their perceptual abilities.

Hyperspecific representation means that information is represented in an extremely detailed and event-specific fashion that minimizes the points of similarity between objects or events. This type of representation reduces the ability to learn perceptual categories requiring complex generalization, generalize perceptual learning, and transfer learning to novel contexts. Effective perceptual categorization is an important precursor to many of the social skills that individuals with ASD have difficulty learning. For instance, part of being able to correctly understand the social cues that guide interactions with others involves learning to correctly categorize facial, vocal, and bodylanguage expressions. This requires recognizing similarities between complex perceptual inputs that vary on a number of dimensions (McCann \& Peppe, 2003; Sasson, 2006; Schwarzer, 2000).

Consistent with theories assuming hyperspecific representation, there is substantial evidence that individuals with ASD have difficulty transferring learning to novel contexts (Mottron \& Burack, 2006), and that perceptual learning generalizes abnormally (Plaisted et al., 1998). However, research examining family resemblance com-

B.A. Church, bchurch@buffalo.edu 
parison in individuals with ASD has produced mixed findings. Family resemblance comparison involves the ability to treat objects as part of the same category on the basis of their overall similarity to other members without any defining features or simple rules to indicate membership. Family resemblance comparison may occur because people create an average representation (or prototype) for the category and compare new examples with that average (e.g., Rosch \& Mervis, 1975), or because people compare new examples with all those previously experienced and then average the similarity from all of the comparisons when categorizing (e.g., Nosofsky, 1984). All categorizing processes involving family resemblance comparison require the ability to assess similarity across multiple representations and, thus, will be negatively impacted by hyperspecific representation.

Some researchers have found abnormalities in the use of family resemblance comparison in children with lowfunctioning ASD when classifying animal-like stimuli (Klinger \& Dawson, 2001), and in children and adults with high-functioning ASD (HFASD) when classifying faces (Gastgeb, Rump, Best, Minshew, \& Strauss, 2009). Other researchers, however, have found that children with HFASD show normal tendencies to use average information about the category when trying to remember or categorize simple line drawings (Molesworth, Bowler, \& Hampton, 2005, 2008). Also, simulations of the performance of adults with HFASD using the general context model (GCM) have been interpreted as evidence against hyperspecific representation, because the individuals with HFASD did not show higher sensitivity scores when final performance was modeled (Bott, Brock, Brockdorff, Boucher, \& Lamberts, 2006). Sensitivity scores are thought to reflect the ability to recognize similarity to learning exemplars, and Bott et al. predicted that hyperspecificity would produce higher sensitivity to the exemplars. Therefore, sensitivity scores similar to those of controls were interpreted as disconfirmation of hyperspecific representation in ASD. However, hyperspecificity should most strongly affect the ability to recognize similarity between items and to average across them. Consequently, it may not be reflected in this overall sensitivity score.

The reasons for these differences across studies are debated. Some suggest that the "difficulties" with family resemblance comparison associated with ASD may actually reflect misunderstandings of the instructions (Molesworth et al., 2008) or problems specific to the processing of social stimuli. Others have argued that the binary feature stimuli used in studies showing "normal" family resemblance comparisons cannot easily differentiate between rule-based and family resemblance strategies (Gastgeb et al., 2009). Furthermore, in Bott et al. (2006), individuals with HFASD had serious difficulties initially learning categories, suggesting that they learned differently, and null results are always difficult to interpret. Overall, past studies have not produced a clear picture of whether individuals with ASD have difficulty generalizing on the basis of perceptual similarity, as predicted by theories as- suming hyperspecific representation. It is clear, however, that further research is needed.

The present study attempted to disambiguate the situation by clearly examining whether children with HFASD have difficulties making family resemblance comparisons. School-age children were used because of the availability of clearly diagnosed and described HFASD populations within this cohort, and because of the possibility that extensions of this work could be used to inform treatment programs designed for this population. An influential family resemblance category-learning task - the dot pattern classification task (e.g., Homa, Cross, Cornell, Goldman, \& Schwartz, 1973; Knowlton \& Squire, 1993; Posner \& Keele, 1968; Smith, 2002) was used to determine whether children with HFASD are less likely to use family resemblance than matched typically developing (TD) children. We chose this particular task because performance is optimized by making decisions based on overall visual similarity across members, and it is difficult to perform well using rules. This task has the added advantage of using abstract stimuli with no social relevance. To directly examine whether the participants seemed to be averaging across their experiences, we used prototype-based formal models of categorization that are standard within the literature (e.g., Smith, Redford, $\&$ Haas, 2008) and that have been shown to characterize performance in this task as well as or better than other models (Smith, 2002). Because prototype models assume a comparison with the average of the category, a breakdown in the prototype model's ability to describe the data suggests a breakdown in the use of information about the average of the category (i.e., family resemblance comparisons). Prototype models assume that people are comparing items with average information about the category; so if individuals with HFASD have hyperspecific representations, these models should fail. The GCM also averages information across the category members, but the richer base of representations for comparison and the greater number of free parameters make it more likely to be able to describe performance even if averaging across members is abnormal. Also, similarity averaging is not a parameter in the GCM, so it is less useful for looking at averaging processes. For these reasons, the prototype model was better suited for addressing our question about family resemblance comparison.

We are not making any assumptions about whether averages are captured within the stored representations themselves in the form of a stored prototype (e.g., Homa et al., 1973; Rosch \& Mervis, 1975; Smith, 2002) or calculated during the decision process (e.g., Hintzman, 1986; Nosofsky, 1984), nor are we claiming that comparisons of family resemblance are always the process by which typical adults and children categorize information. There is ample evidence that performance on many tasks is better described by the use of other strategies (e.g., Ashby \& Maddox, 2005; Johansen \& Palmeri, 2002; Minda, Desroches, \& Church, 2008). Our goal is to determine whether children with HFASD use family resemblance comparisons to the same degree as TD children in a task 
Table 1

Demographic Characteristics

\begin{tabular}{|c|c|c|c|c|c|c|}
\hline \multirow[b]{2}{*}{ Characteristic } & \multicolumn{2}{|c|}{$\operatorname{HFASD}(n=20)$} & \multicolumn{2}{|c|}{ Control $(n=20)$} & \multicolumn{2}{|c|}{ Overall Sample $(n=20)$} \\
\hline & $M$ & $S D$ & $M$ & $S D$ & $M$ & $S D$ \\
\hline Age (years) & 9.40 & 1.76 & 9.50 & 1.64 & 9.45 & 1.68 \\
\hline Parent education (years) & 15.43 & 1.83 & 15.65 & 1.95 & 15.54 & 1.87 \\
\hline WISC-IV Short Form IQ & 107.93 & 10.78 & 111.47 & 9.58 & 109.70 & 10.22 \\
\hline \multicolumn{7}{|l|}{ CASL } \\
\hline Expressive Language & 101.75 & 7.33 & - & & & \\
\hline Receptive Language & 109.35 & 13.66 & - & & & \\
\hline \multicolumn{7}{|l|}{ ADI-R } \\
\hline QARSI & 19.05 & 5.75 & & & & \\
\hline QAC & 14.05 & 5.35 & & & & \\
\hline \multirow[t]{2}{*}{ RRSB } & 6.60 & 1.85 & & & & \\
\hline & \multicolumn{2}{|c|}{$n(\%$ of total $)$} & \multicolumn{2}{|c|}{$n(\%$ of total $)$} & \multicolumn{2}{|c|}{$n(\%$ of total $)$} \\
\hline \multirow[t]{2}{*}{ Gender } & \multirow{2}{*}{\multicolumn{2}{|c|}{$\begin{array}{l}\text { Male }=18(90.0) \\
\text { Female }=2(10.0)\end{array}$}} & \multirow{2}{*}{\multicolumn{2}{|c|}{$\begin{array}{l}\text { Male }=18(90.0) \\
\text { Female }=2(10.0)\end{array}$}} & \multicolumn{2}{|c|}{ Male $=36(90.0)$} \\
\hline & & & & & \multicolumn{2}{|c|}{ Female = $4(10.0)$} \\
\hline \multirow[t]{3}{*}{ Ethnicity } & \multicolumn{2}{|c|}{ Caucasian $=17(85.0)$} & \multicolumn{2}{|c|}{ Caucasian $=18(90.0)$} & \multirow{2}{*}{\multicolumn{2}{|c|}{$\begin{array}{l}\text { Caucasian }=35(87.5) \\
\text { African American }=2(5.0)\end{array}$}} \\
\hline & \multicolumn{2}{|c|}{ African American $=1(5.0)$} & \multicolumn{2}{|c|}{ African American $=1(5.0)$} & & \\
\hline & \multicolumn{2}{|c|}{ Biracial $=2(10.0)$} & \multicolumn{2}{|c|}{ Biracial $=1(5.0)$} & \multicolumn{2}{|c|}{ Biracial $=3(7.5)$} \\
\hline \multirow{3}{*}{ Diagnosis } & \multicolumn{6}{|c|}{$\mathrm{AD}=15(75.0)$} \\
\hline & \multicolumn{6}{|c|}{ PDDNOS $=4(20.0)$} \\
\hline & \multicolumn{6}{|c|}{$\mathrm{HFA}=1(5.0)$} \\
\hline
\end{tabular}

Note-Diagnostic categories reported for the HFASD group in this table constitute diagnoses made by external clinicians (i.e., contained in reports submitted by parents). AD, Asperger's Disorder; PDDNOS, Pervasive Developmental Disorder-Not Otherwise Specified; HFA, Autism (highfunctioning); WISC-IV, Wechsler Intelligence Scale for Children-4th Edition; CASL, Comprehensive Assessment of Spoken Language; ADI-R, Autism Diagnostic Interview-Revised; QARSI, Qualitative Abnormalities in Reciprocal Social Interactions; QAC, Qualitative Abnormalities in Communication; RRSB, Restricted, Repetitive, and Stereotyped Patterns of Behavior. CASL and ADI-R data collected only for children in the HFASD group as a screening measure for the social treatment study. All testing to determine inclusion in the social treatment study from which the HFASD sample was recruited (i.e., WISC-IV, CASL, and ADI-R) was done by members of the research team.

where the stimuli make these types of comparison maximally helpful.

\section{METHOD}

\section{Participants}

Twenty children with HFASD and 20 TD children, ages 7 to 12 years, participated (see Table 1). The HFASD and TD groups were matched on age, gender, and IQ. Statistical analyses showed that there were no significant differences between the groups for age $[t(38)=0.091, p=.928]$, parent education $[t(38)=-0.696, p=$ $.491]$, short-form IQ $[t(38)=-1.148, p=.258]$, ethnicity (Fisher's exact test $p=1.00$; Pearson chi-square $p=.633$ ), or gender (Fisher's exact test $p=1.00$; Pearson chi-square $p=1.00$ ).

Children in the HFASD group were recruited from a summer social treatment study involving children with HFASD, and all had met specific inclusion criteria, including WISC-IV (Wechsler, 2003) short-form IQ composite $>70$ and a major index score (i.e., VCI or PRI) $\geq 80$; receptive or expressive language score $\geq 80$ (CASL short form; Carrow-Woolfolk, 1999); and a score meeting ASD criteria on the Autism Diagnostic Interview-Revised (ADI-R; Rutter, LeCouteur, \& Lord, 2003).

TD children were recruited from the community via flyers. Exclusionary criteria included any diagnosis of a psychiatric disorder or a history of receiving special education services. Parents of the TD children completed a background form, and the children completed the WISC-IV short form.

\section{Stimuli and Apparatus}

A randomly generated prototype shape, 40 distortions from the prototype varying in level of distortion, with higher level numbers denoting greater distortion $(5 \mathrm{~L}-2,10 \mathrm{~L}-3,5 \mathrm{~L}-4,10 \mathrm{~L}-5$, and $10 \mathrm{~L}-7$ ), and 45 randomly generated noncategory members were created (see the online Appendix for construction methods and stimuli). The randomly generated shapes were visually inspected by the researchers to exclude and replace any that seemed similar to category members (none were excluded). Colors of medium brightness were randomly assigned to the shapes to make the task more interesting to children (Lawson, 2003). Each shape was presented in the center of the screen against a black background, and two icons were presented at the top on either side of the screen (a gray cave icon in the top right corner and a red circle with a line through it in the top left corner).

Participants were tested using IBM-compatible computers and headphones. DMDX experimental software was used to present stimuli and feedback and collect responses (Forster \& Forster, 2003).

\section{Design and Procedure}

A $2 \times 7$ mixed factorial design was used with category endorsement (calling the item a "cave ghost") as the dependent measure. Diagnosis (HFASD vs. TD) and stimulus type (prototype, L-2, L-3, L-4, L-5, L-7, and random) served as the independent variables.

The HFASD and TD participants completed the same task. The training phase consisted of $5 \mathrm{~L}-3,5 \mathrm{~L}-5$, and $5 \mathrm{~L}-7$ category stimuli and 15 random stimuli, for a total of 30 trials. The testing phase consisted of five examples of each of six category stimulus types (prototype, L-2, L-3, L-4, L-5, and L-7) and 30 random stimuli, for a total of 60 trials. None of the stimuli from the training phase were reused during the testing phase, and stimuli were presented in a fixed random order. The trials were self-paced, and the shape stayed on the screen until the child responded. If a child did not respond within $20 \mathrm{sec}$, this was counted as a missing value, and the next trial began.

Children were asked to find the "cave ghosts" (shapes belonging to the category) and were informed that during the first part of the game, they would receive feedback to help them learn what cave ghosts look like. If the child chose correctly, a dancing monkey appeared. If the child was incorrect, the to-be-categorized shape moved to the icon at the top of the screen corresponding to the correct answer (see Figure 1). The cave icon corresponded to the response button used to indicate that the shape was a cave ghost, and a red circle with a line through it corresponded to the response button used to indicate that it was not a cave ghost. Children were told that cave 


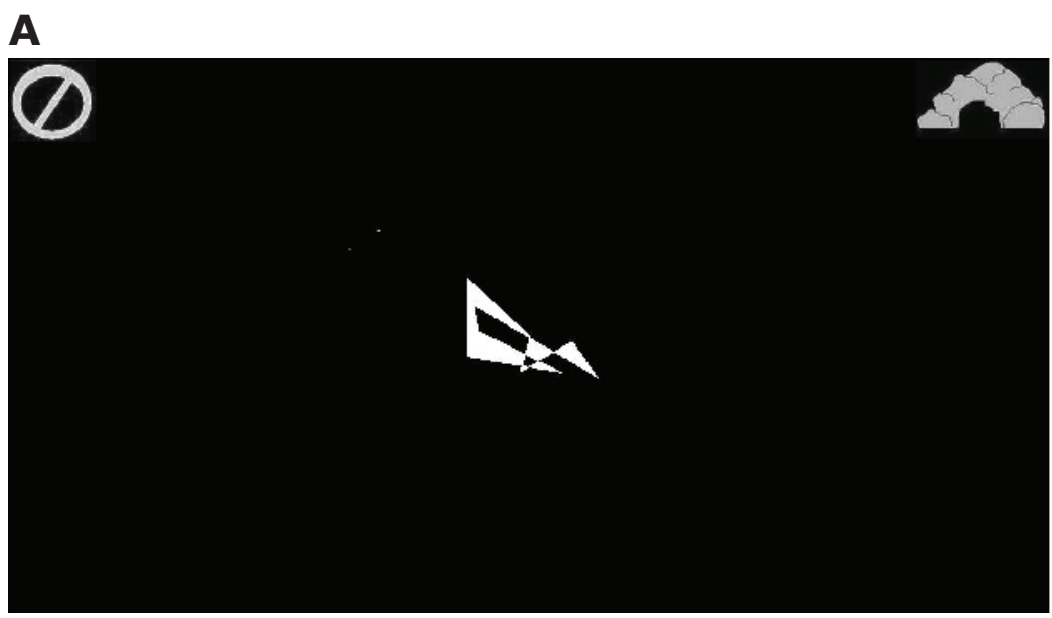

B

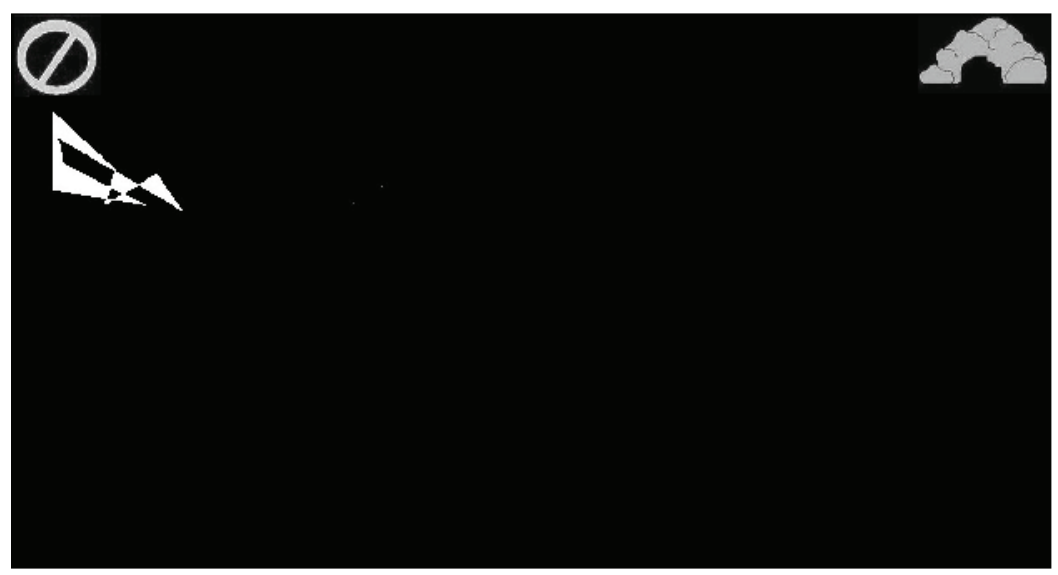

Figure 1. (A) Example of a categorization trial. (B) Example of a correction trial after an incorrect answer during training.

ghosts looked similar, and that shapes that looked different were not cave ghosts. This was done to maximize the likelihood that the children would focus on the overall similarity (family resemblance) and not look for rules. They were also told that cave ghosts could come in any color, so they should not base their decisions on color. Inspection of the children's responses suggested that all the children were able to follow this instruction. During the test phase, they were informed that they would not receive feedback.

\section{RESULTS}

Figure 2 depicts the average of the observed endorsements (saying it was a cave ghost) by stimulus type and the average of the best-fitting model-predicted endorsement profiles for the TD and HFASD groups separately. All statistical comparisons were two-tailed and used an $\alpha$ of .05. Because of the greater variability of performance for the HFASD group, $t$ tests appropriate for groups with unequal variance were used. Analyses of the categorization test data showed that the TD group performed significantly better $[t(28)=3.636, p<.001$, Cohen's $d=$ $1.141]$ (TD, $M=89.1 \%$ correct, $S D=9.9 \%$; HFASD, $M=71.6 \%$ correct, $S D=19.3 \%$ ). Analyses were also conducted to examine the pattern of endorsement across different stimulus types. A $2 \times 7$ ANOVA of the percentage of endorsement, using group as the betweenparticipants and stimulus type as the within-participant variables, showed significant main effects of both group $\left[F(1,38)=8.598, p<.01, \eta_{\mathrm{p}}^{2}=.185\right]$ and stimulus type $\left[F(6,228)=63.108, p<.001, \eta_{\mathrm{p}}^{2}=.624\right]$, showing that TD children generally endorsed the category more often and that different stimulus types were endorsed differently. A significant interaction between stimulus type and group $\left[F(6,228)=6.076, p<.001, \eta_{\mathrm{p}}^{2}=.135\right]$ also indicated that the pattern of endorsement across stimulus types was different for the two groups. Post hoc analyses using Bonferroni correction showed that children with HFASD endorsed the prototype and L-3 distortions significantly less $[t(24)=3.601, p<.005$, Cohen's $d=$ $1.132 ; t(24)=3.38, p<.005$, Cohen's $d=1.066]$ and the random items significantly more $[t(33)=3.145, p<$ .005 , Cohen's $d=0.993]$ than the TD children. As can be seen in Figure 2, unlike the TD group, the HFASD group endorsed the prototype numerically less than distortions, though this difference was significant only for L-2 distortions $[t(19)=3.269, p<.005$, Cohen's $d=0.451$; all other $t \mathrm{~s}<1]$. Further analyses showed that $60 \%$ of the 

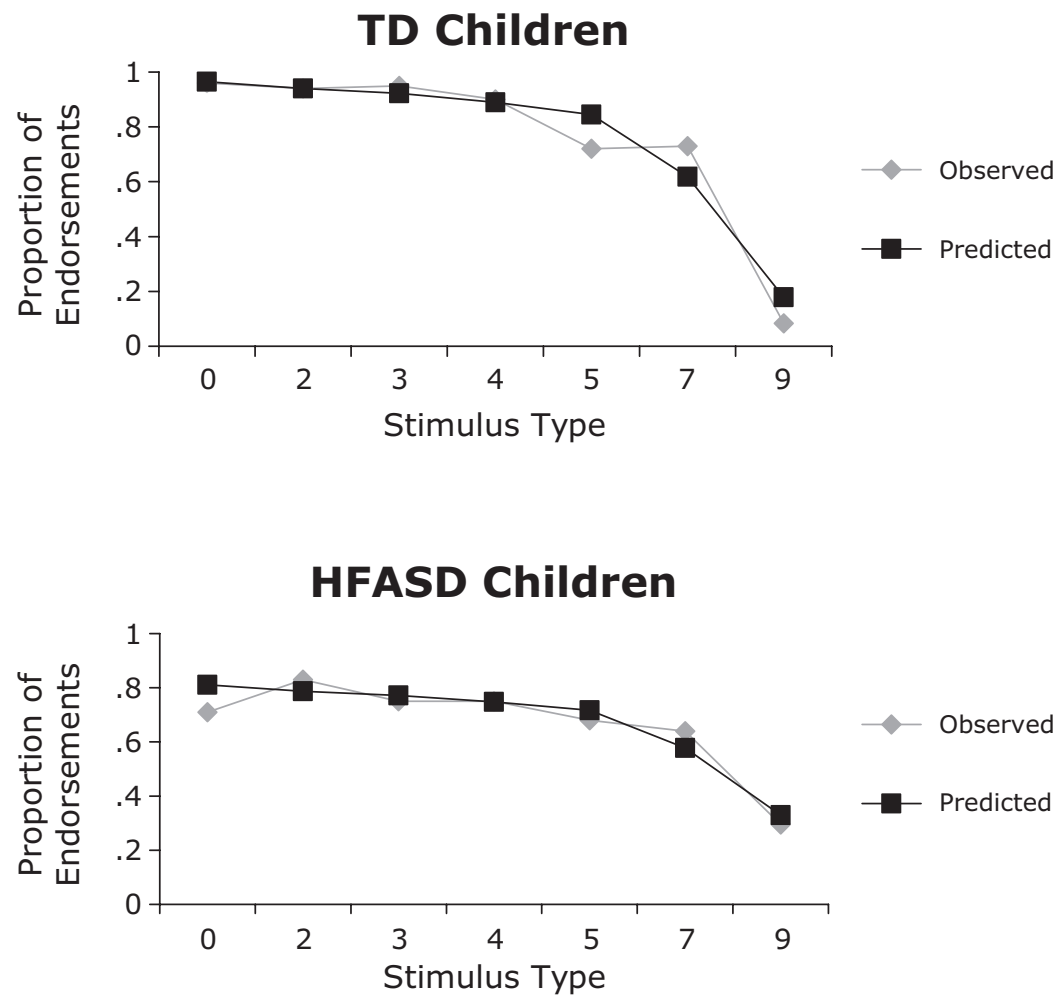

Figure 2. Observed versus model-predicted endorsements for typically developing (TD) and high-functioning autism spectrum disorder (HFASD) groups. $0=$ prototype, $2=\mathrm{L}-2$ distortion, $3=\mathrm{L}-3$ distortion, $4=\mathrm{L}-4$ distortion, $5=\mathrm{L}-5$ distortion, $7=\mathrm{L}-7$ distortion, and $9=$ random patterns.

children with HFASD endorsed the prototype less than the members of at least one other distortion category, whereas this was true for only $15 \%$ of the TD children.

To further understand this interaction, we examined the fit of a standard prototype model of categorization to each individual's performance. This model assumes that participants make category decisions by comparing to-be-categorized items with the prototype. Similarity is computed, and a decision is made. There are two free parameters in the model that can vary for each participant. The first is criterion (c), representing the general similarity between the items and members of other categories in the world. The second, sensitivity $(k)$, is a measure of the participant's sensitivity to perceptual distance from the prototype. The model uses a hill-climbing algorithm to find the best-fitting parameters and then computes the model's fit to the data by determining the sum of the squared deviations $(S S D)$ between the observed and predicted data (model specifics and algorithms in the online Appendix). Larger $S S D$ scores denote a worse fit. The model let us determine the extent to which participants' pattern of endorsement across distortion levels was consistent with making judgments on the basis of the central tendency of the category. Table 2 presents the average fit (SSD), sensitivity (c), and criterion $(k)$ parameter values for each group. Significant fit differences were found $[t(24)=2.059, p=.05$, Cohen's $d=0.649$ ], indicating that TD children showed a better fit to the model than children with HFASD. TD children also demonstrated significantly higher estimated sensitivityparameter scores $[t(29)=3.572, p<.002$, Cohen's $d=$ $1.127]$ and significantly lower mean criterion-parameter values $[t(27)=3.377, p<.003$, Cohen's $d=0.8]$. These differing parameter estimates suggest that TD children resolved distances from the category prototype more sensitively. However, parameter values from models that fit poorly should be interpreted cautiously.

To explore whether the differences between groups were produced by a small subset of the children with HFASD, an additional examination was done of the percentage of children in each group with a good fit to the model $(S S D<.1)$, or any sensitivity to distance from the prototype $(c>1)$. A clear majority of TD children fit the model well $(60 \%)$ and were sensitive to the distance from the prototype (95\%). Conversely, only a minority of children with HFASD conformed well to the prototype model $(35 \%)$, and only slightly more than half demonstrated any

Table 2

Prototype Model Measures (Means and Standard Deviations)

\begin{tabular}{|c|c|c|c|c|c|c|}
\hline \multirow{2}{*}{$\begin{array}{c}\text { Participant } \\
\text { Diagnosis }\end{array}$} & \multicolumn{2}{|c|}{ Sensitivity $(c)$} & \multicolumn{2}{|c|}{ Criterion $(k)$} & \multicolumn{2}{|c|}{ Fit $(S S D)$} \\
\hline & $M$ & $S D$ & $M$ & $S D$ & $M$ & $S D$ \\
\hline HFASD & 1.205 & 1.080 & 0.29 & 0.305 & 0.171 & 0.152 \\
\hline TD & 2.176 & 0.564 & 0.047 & 0.132 & 0.097 & 0.053 \\
\hline
\end{tabular}

Note-HFASD, high-functioning autism spectrum disorder; TD, typically developing. 
sensitivity (55\%). Lastly, in the HFASD sample, there was a strong positive correlation between sensitivity and overall task performance $(r=.968)$, suggesting that sensitivity to distance from the category average predicted performance on this task The correlation between fit and performance, although significant, was less impressive $(r=-.51)$, indicating that the fit of the model was not largely determined by correct performance. Further analyses showed no significant Bonferroni-corrected correlations between participant's IQ (verbal or short form) and the measures of fit and sensitivity in either group (all $r \mathrm{~s}<.3$ ) and nonsignificant correlations between fit and sensitivity and ADI-R scores on each of the subscales (all $r \mathrm{~s}<.6$ ).

\section{DISCUSSION}

This study tested the prediction that hyperspecific representation in ASD leads to less use of family resemblance. Consistent with hyperspecificity, we found that children with HFASD showed significantly less endorsement of the prototype of the category, seemingly less sensitivity to the distance between new items and the average of the category, and significantly poorer fit to a model that assumes that they are making comparisons to that average. The children with HFASD were even less likely to endorse the prototype than other high-level distortion category members. These results strongly suggest that many children with HFASD are not using information about overall average similarity across members (family resemblance) when making categorization decisions. These differences could reflect an inability to form average (prototype) representations, an inability to properly judge or sum similarity across many comparisons, or a bias against using family resemblance comparison. Consistent with many of the cognitive-perceptual theories of autism (Happe \& Frith, 2006; Mottron et al., 2006; Plaisted, 2001), any of these processes would be disrupted if individuals with ASD form hyperspecific representations that cannot be easily averaged.

While the present findings are consistent with recent work on categorization of faces (Gastgeb et al., 2009), they also extend those findings by showing that this is a general categorization problem and not specific to social stimuli. Individuals with HFASD do not effectively make family resemblance comparisons even when the stimuli have no social implications. Stimuli with complex dimensions of similarity (like morphed faces or Posner shapes) may be more problematic for individuals with ASD than are stimuli made up of simple binary feature combinations (Bott et al., 2006; Molesworth et al., 2005, 2008). Many natural categories have these complex similarity relationships, including the social categories that create problems for individuals with ASD (e.g., facial emotion, facial recognition, body language, prosody).

Taken together with previous findings on categorical processing (Bott et al., 2006; Gastgeb et al., 2009; Klinger \& Dawson, 2001; Molesworth et al., 2005, 2008), two things become clear. First, even high-functioning children with ASD show difficulties using family resemblance in categorization. Second, carefully identifying how categorization processes in children with ASD differ from those in typically developing children is necessary to fully understand the social and cognitive difficulties in this population.

\section{AUTHOR NOTE}

This research was supported in part by grants from the National Institute of Mental Health (Grant MH 67952), the NSF (Grant SBE 0542013 to the Temporal Dynamics of Learning Center), and the John R. Oishei Foundation. We thank Justin Couchman, Joe Boomer, Kim Uminski, Estella Liu, J. David Smith, and the editor and reviewers for help with stimulus construction, data collection and analyses, and useful commentary, respectively. Correspondence concerning this article should be addressed to B. A. Church, Department of Psychology, Park Hall, University at Buffalo, State University of New York, Buffalo, NY 14260 (e-mail: bchurch@buffalo.edu).

\section{Note-Accepted by Cathleen M. Moore's editorial team.}

\section{REFERENCES}

Ashby, F. G., \& Maddox, W. T. (2005). Human category learning. Annual Review of Psychology, 56, 149-178.

Bott, L., Brock, J., BrockdorfF, N., Boucher, J., \& Lamberts, K. (2006). Perceptual similarity in autism. Quarterly Journal of Experimental Psychology, 59, 1237-1254.

CARrow-Woolfolk, E. (1999). Comprehension assessment of spoken language. Circle Pines, MN: American Guidance Service.

Forster, K. L., \& Forster, J. C. (2003). DMDX: A windows display program with millisecond accuracy. Behavior Research Methods, Instruments, \& Computers, 35, 116-124.

Gastgeb, H. Z., Rump, K. M., Best, C. A., Minshew, N. J., \& Strauss, M. S. (2009). Prototype formation in autism: Can individuals with autism abstract facial prototypes? Autism Research, 2, 279-284.

Happe, F., \& Frith, U. (2006). The weak coherence account: Detailfocused cognitive style in autism spectrum disorders. Journal of Autism \& Developmental Disorders, 36, 5-25.

HintZman, D. L. (1986). "Schema abstraction" in a multiple-trace memory model. Psychological Review, 93, 411-428.

Homa, D., Cross, J., Cornell, D., Goldman, D., \& Schwartz, S. (1973). Prototype abstraction and classification of new instances as a function of number of instances defining the prototype. Journal of Experimental Psychology, 101, 116-122.

IAROCCI, G., \& MCDonaLD, J. (2006). Sensory integration and the perceptual experience of persons with autism. Journal of Autism \& Developmental Disorders, 36, 77-90.

Johansen, M. K., \& Palmeri, T. J. (2002). Are there representational shifts during category learning? Cognitive Psychology, 45, 482-553.

Just, M. A., Cherkassky, V. L., Keller, T. A., \& Minshew, N. J. (2004). Cortical activation and synchronization during sentence comprehension in high-functioning autism: Evidence of underconnectivity. Brain, 127, 1811-1821.

KLinger, L. G., \& Dawson, G. (2001). Prototype formation in autism. Development \& Psychopathology, 13, 111-124.

KNowlton, B. J., \& SQuire, L. R. (1993). The learning of categories: Parallel brain systems for item memory and category knowledge. Science, 262, 1747-1749.

Lawson, J. (2003). Depth accessibility difficulties: An alternative conceptualization of autism spectrum conditions. Journal for the Theory of Social Behavior, 33, 189-202.

McCann, J., \& PepPe, S. (2003). Prosody in autism spectrum disorders. International Journal of Language \& Communication Disorders, $\mathbf{3 8}$, 325-350.

MCClelland, J. L. (2000). The basis of hyperspecificity in autism: A preliminary suggestion based on properties of neural nets. Journal of Autism \& Developmental Disorders, 30, 497-502.

Minda, J. P., Desroches, A. S., \& Church, B. A. (2008). Learning rule-described and non-rule-described categories: A comparison of 
children and adults. Journal of Experimental Psychology: Learning, Memory, \& Cognition, 34, 1518-1533.

Molesworth, C. J., Bowler, D. M., \& Hampton, J. A. (2005). The prototype effect in recognition memory: Intact in autism? Journal of Child Psychology \& Psychiatry, 46, 661-672.

Molesworth, C. J., Bowler, D. M., \& Hampton, J. A. (2008). When prototypes are not best: Judgments made by children with autism. Journal of Autism \& Developmental Disorders, 38, 1721-1730.

Mottron, L., \& Burack, J. (2006). Autism: A different perception. Journal of Autism \& Developmental Disorders, 36, 1-3.

Mottron, L., Dawson, M., Soulieres, I., Hubert, B., \& Burack, J. (2006). Enhanced perceptual functioning in autism: An update, and eight principles of autistic perception. Journal of Autism \& Developmental Disorders, 36, 27-43.

Nosofsky, R. M. (1984). Choice, similarity, and the context theory of classification. Journal of Experimental Psychology: Learning, Memory, \& Cognition, 10, 104-114.

O'Riordan, M. A., \& Plaisted, K. C. (2001). Enhanced discrimination in autism. Quarterly Journal of Experimental Psychology, 54A, 961-979.

PLAISTED, K. C. (2001). Reduced generalization in autism: An alternative to weak central coherence. In J. A. Burack, T. Charman, N. Yirmiya, \& P. R. Zelazo (Eds.), The development of autism: Perspectives from theories and research (pp. 149-169). Mahwah, NJ: Erlbaum.

Plaisted, K. C., O'Riordan, M. A., \& Baron-Cohen, S. (1998). Enhanced discrimination of novel highly similar stimuli by adults with autism during a perceptual learning task. Journal of Child Psychology \& Psychiatry, 39, 765-775.
Posner, M. I., \& Keele, S. W. (1968). On the genesis of abstract ideas. Journal of Experimental Psychology, 77, 353-363.

Rosch, E., \& Mervis, C. B. (1975). Family resemblances: Studies in the internal structure of categories. Cognitive Psychology, 7, 573-605.

Rutter, M., LeCouteur, A., \& Lord, C. (2003). The Autism Diagnostic Interview-Revised $(A D I-R)$. Los Angeles: Western Psychological Services.

SAsson, N. J. (2006). The development of face processing in autism. Journal of Autism \& Developmental Disorders, 36, 381-394.

SCHWARZER, G. (2000). Development of face processing: The effect of face inversion. Child Development, 71, 391-401.

Sмiтh, J. D. (2002). Exemplar theory's predicted typicality gradient can be tested and disconfirmed. Psychological Science, 13, 437-442.

Smith, J. D., Redford, J. S., \& HaAs, S. M. (2008). Prototype abstraction by monkeys (Macaca mulatta). Journal of Experimental Psychology: General, 137, 390-401.

WeCHSLER, D. (2003). Wechsler Intelligence Scale for Children (4th ed.). San Antonio: Psychological Corp.

\section{SUPPLEMENTAL MATERIALS}

An appendix containing stimuli, detailed descriptions of stimulus construction methods, and model specifics and algorithms may be downloaded from http://pbr.psychonomic-journals.org/content/supplemental.

(Manuscript received May 12, 2010;

revision accepted for publication August 8, 2010.) 